\title{
Galactic bulge population II Cepheids in the VVV survey: period-luminosity relations and a distance to the Galactic centre ${ }^{\star}$
}

\author{
A. Bhardwaj ${ }^{1,2}$, M. Rejkuba ${ }^{1,3}$, D. Minniti ${ }^{4,5,6}$, F. Surot $^{1}$, E. Valenti ${ }^{1}$, M. Zoccali ${ }^{7,5}$, \\ O. A. Gonzalez ${ }^{8}$, M. Romaniello ${ }^{1,3}$, S. M. Kanbur ${ }^{9}$, and H. P. Singh ${ }^{2}$ \\ ${ }^{1}$ European Southern Observatory, Karl-Schwarzschild-Straße 2, 85748 Garching, Germany \\ e-mail: anupam.bhardwajj@gmail.com; abhardwaj@eso.org \\ 2 Department of Physics and Astrophysics, University of Delhi, Delhi 110007, India \\ 3 Excellence Cluster Universe, Boltzmann-Str. 2, 85748 Garching bei München, Germany \\ ${ }^{4}$ Departamento de Física, Facultad de Ciencias Exactas, Universidad Andrés Bello, Av. Fernandez Concha 700, Las Condes, \\ Santiago, Chile \\ 5 Instituto Milenio de Astrofisica, 1515 Las Condes, Santiago, Chile \\ 6 Vatican Observatory, 00120 Vatican City State, Italy \\ 7 Pontificia Universidad Católica de Chile, Instituto de Astrofísica, Av. Vicuña Mackenna 4860, Santiago, Chile \\ 8 UK Astronomy Technology Centre, Royal Observatory, Blackford Hill, Edinburgh EH9 3HJ, UK \\ 9 State University of New York, Oswego, NY 13126, USA
}

Received 21 March 2017 / Accepted 12 July 2017

\section{ABSTRACT}

\begin{abstract}
Context. Multiple stellar populations of different ages and metallicities reside in the Galactic bulge that trace its structure and provide clues to its formation and evolution.

Aims. We present the near-infrared observations of population II Cepheids in the Galactic bulge from VISTA Variables in the Vía Láctea (VVV) survey. The $J H K_{\text {s }}$ photometry together with optical data from Optical Gravitational Lensing Experiment (OGLE) survey provide an independent estimate of the distance to the Galactic centre. The old, metal-poor and low-mass population II Cepheids are also investigated as useful tracers for the structure of the Galactic bulge.

Methods. We identify 340 population II Cepheids in the VVV survey Galactic bulge catalogue based on their match with the OGLE-III Catalogue. The single-epoch $J H$ and multi-epoch $K_{\mathrm{s}}$ observations complement the accurate periods and optical $(V I)$ mean-magnitudes from OGLE. The sample consisting of BL Herculis and W Virginis subtypes is used to derive period-luminosity relations after correcting mean-magnitudes for the extinction. Our $K_{\mathrm{s}}$-band period-luminosity relation, $K_{\mathrm{s}}=-2.189(0.056)[\log (P)-1]+11.187(0.032)$, is consistent with published work for BL Herculis and W Virginis variables in the Large Magellanic Cloud.

Results. We present a combined OGLE-III and VVV catalogue with periods, classification, mean magnitudes, and extinction for 264 Galactic bulge population II Cepheids that have good-quality $K_{\mathrm{s}}$-band light curves. The absolute magnitudes for population II Cepheids and RR Lyraes calibrated using Gaia and Hubble Space Telescope parallaxes, together with calibrated magnitudes for Large Magellanic Cloud population II Cepheids, are used to obtain a distance to the Galactic centre, $R_{0}=8.34 \pm 0.03$ (stat.) \pm 0.41 (syst.), which changes by ${ }_{-0.25}^{+0.05}$ with different extinction laws. While noting the limitation of small number statistics, we find that the present sample of population II Cepheids in the Galactic bulge shows a nearly spheroidal spatial distribution, similar to metal-poor RR Lyrae variables. We do not find evidence of the inclined bar as traced by the metal-rich red-clump stars.

Conclusions. Population II Cepheid and RR Lyrae variables follow similar period-luminosity relations and trace the same metal-poor old population in the Galactic bulge. The number density for population II Cepheids is more limited as compared to abundant RR Lyraes but they are bright and exhibit a wide range in period that provides a robust period-luminosity relation for an accurate estimate of the distance to the Galactic centre.
\end{abstract}

Key words. stars: variables: Cepheids - Galaxy: bulge - Galaxy: structure - galaxies: Magellanic Clouds - distance scale

\section{Introduction}

Population II Cepheids are low-mass, metal-poor stars that are found in globular clusters, Galactic disc, and bulge populations (Wallerstein 2002; Sandage \& Tammann 2006). These Type II Cepheid (T2C) variables are more than a magnitude fainter than Classical or Type I Cepheids with similar periods and follow a shallower period-luminosity relation (PLR or "Leavitt Law", Leavitt \& Pickering 1912). T2Cs reside in the instability strip just above (brighter) RR Lyrae (RRL) variables and

\footnotetext{
* The full Table 1 is only available at the CDS via anonymous ftp to cdsarc.u-strasbg.fr (130.79.128.5) or via

http://cdsarc.u-strasbg.fr/viz-bin/qcat?J/A+A/605/A100
}

are subdivided into three subclasses, BL Herculis (BLH), W Virginis (WVR), and RV Tauris (RVT). These subclasses represent different evolutionary states: short-period BLH move from blue horizontal branch to asymptotic giant branch (AGB), intermediate period WVR stars undergo helium shell flashes and make temporary excursions from the AGB into the instability strip, while long period RVT suggest post-AGB evolution (Wallerstein 2002). The characteristic light curves for the subclasses of T2Cs are different and the PLRs exhibit a greater dispersion and non-linearity at optical wavelengths (Nemec et al. 1994; Alcock et al. 1998; Kubiak \& Udalski 2003; Majaess et al. 2009; Schmidt et al. 2009, and references within), thus limiting their use as primary distance indicators. At 
near-infrared (NIR) wavelengths, T2Cs and RRLs follow similar PLRs (Sollima et al. 2006; Matsunaga et al. 2006; Ripepi et al. 2015; Bhardwaj et al. 2017) and because T2Cs are relatively bright variables they can be used to obtain robust distances. These variables provide an independent method to determine the distance to the Galactic centre and to trace the structure of the old stellar population in the Galactic bulge. For example, Groenewegen et al. (2008) estimated a T2C and RRL based distance to the Galactic centre: $R_{0}=7.94 \pm 0.37 \mathrm{kpc}$.

The optical light curves of T2Cs in the Galactic bulge and the Magellanic Clouds (MC) are provided by the third phase of the Optical Gravitational Lensing Experiment (OGLE-III) survey (Soszyński et al. 2008, 2010, 2011). The NIR studies of these variables in the MC were carried out by Matsunaga et al. (2009, 2011), Ciechanowska et al. (2010), Ripepi et al. (2015), and Bhardwaj et al. (2017). T2Cs in the Galactic globular clusters at $J H K_{\mathrm{s}}$ wavelengths were observed by Matsunaga et al. (2006). These authors discussed the T2C PLRs and their distance scale applications at NIR wavelengths. The time-series NIR observations for T2Cs in the Galactic bulge have been limited to a sample of 39 Cepheids observed by Groenewegen et al. (2008).

The VISTA Variables in the Vía Láctea (VVV) survey (Minniti et al. 2010) has provided a large amount of variable star data to probe the 3D structure of the Galactic bulge (Wegg \& Gerhard 2013; Gonzalez et al. 2013; Valenti et al. 2016; Zoccali \& Valenti 2016, and reference therein). Thanks to high-resolution spectroscopic investigations of a sizeable sample of bulge giants (Zoccali et al. 2008; Hill et al. 2011; Ness et al. 2013; Rojas-Arriagada et al. 2014; Gonzalez et al. 2015a, and references therein) it is now widely accepted that the bulge metallicity distribution is broad (i.e. $-1 \leq[\mathrm{Fe} / \mathrm{H}] \leq+0.5 \mathrm{dex}$ ), bimodal, and with two peaks a few dex below and above the solar value. The observed metallicity gradient as a function of the height from the Galactic plane is due to the change of the relative fraction of the metal-rich and metal-poor components along the line of sight. On the other hand, the vast majority of the photometric studies aimed at dating the bulge stars (Ortolani et al. 1995; Kuijken \& Rich 2002; Zoccali et al. 2003; Sahu et al. 2006; Clarkson et al. 2008, 2011; Valenti et al. 2013) revealed a predominantly old stellar population. However, according to spectroscopic microlensing follow-up (Bensby et al. 2017, and references therein) a smaller but significant fraction of young and intermediate age population may also be present. The old metal-poor tracers of the bulge show an axisymmetric and spheroidal distribution (RRLs, Miras, red-clump giants, Dékány et al. 2013; Catchpole et al. 2016; Gran et al. 2016; Zoccali et al. 2017), or a triaxial ellipsoidal distribution (RRLs, Pietrukowicz et al. 2015). The metal-rich red-clump giants trace the X-shaped bar in the bulge (McWilliam \& Zoccali 2010; Nataf et al. 2010; Saito et al. 2011; Gonzalez et al. 2015b; Zoccali et al. 2017).

Recently, Minniti et al. (2016) discovered a dozen fundamental-mode RRL variables in the vicinity of the Galactic centre. T2Cs are less abundant but brighter than RRL and, therefore, are easy to find in highly extincted regions in the bulge. A search for new T2Cs in the whole VVV bulge area will be presented elsewhere. For the present analysis, we use the sample that has optical counterparts in the OGLE catalogue.

The paper is structured as follows: we discuss photometry of T2Cs from the VVV survey in Sect. 2. We derive PLRs at $J H K_{\mathrm{s}}$ wavelengths and compare our results with published work in Sect. 3. We determine a distance to the Galactic centre using calibrated absolute magnitudes for T2Cs in Sect. 4. In Sect. 5, we discuss the spatial distribution of T2Cs in the Galactic bulge and
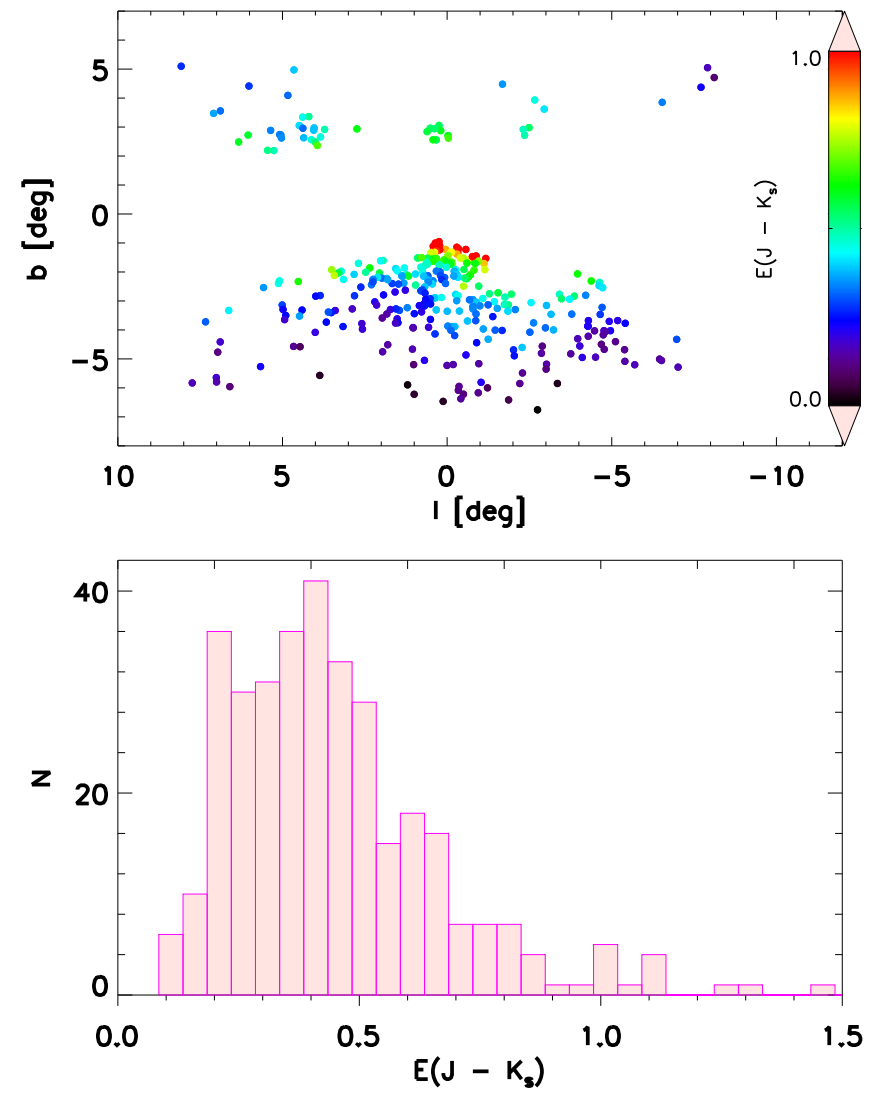

Fig. 1. Top panel: spatial distribution and $E\left(J-K_{\mathrm{s}}\right)$ colour excess for Type II Cepheids in VVV survey. Bottom panel: histogram of the $E(J-$ $K_{\mathrm{s}}$ ) colour excess. Using reddening law of Nishiyama et al. (2009), the extinction in $K_{\mathrm{s}}$-band amounts to, $A_{K_{\mathrm{s}}}=0.528 E\left(J-K_{\mathrm{s}}\right)$.

compare our results with RRLs and red-clump stars. We summarize our results in Sect. 6.

\section{The data}

We present NIR photometry of T2Cs from the VVV survey (Minniti et al. 2010; Saito et al. 2012) latest data release (DR4) catalogue (Hempel et al., in prep.). We perform a cross-match of positions for the OGLE-III bulge T2C sample to this catalogue within a $1^{\prime \prime}$ search radius to identify 340 Cepheids. The median separation between OGLE and VVV sources is $0.08^{\prime \prime}$ with a standard deviation of $0.15^{\prime \prime}$ and more than $95 \%$ of the matched sources have a separation of $\lesssim 0.5^{\prime \prime}$. We adopt the classification based on I-band light curves from OGLE-III and there are $147 \mathrm{BLH}, 123 \mathrm{WVR}$, and 70 RVT type variables with $J H K_{\mathrm{s}}$ observations.

The VVV $J H$-band magnitudes are single epoch observations while the $K_{\mathrm{s}}$-band has multi-epoch data. The number of observations in $K_{\mathrm{s}}$ varies from approximately ten to $\sim 100$ depending on the location and brightness of the $\mathrm{T} 2 \mathrm{C}$, and on average there are $\sim 50$ epochs per light curve. The typical apparent magnitudes for T2Cs in $K_{\mathrm{s}}$ range from $\sim 15$ to $\sim 10 \mathrm{mag}$. The period $(P)$, time of maximum brightness in $I$-band, and the optical $(V I)$ mean-magnitudes for the matched T2Cs are taken from the OGLE-III catalogue (Soszyński et al. 2011). The $E\left(J-K_{\mathrm{s}}\right)$ colour excess for T2Cs in the bulge is obtained using the extinction maps of Gonzalez et al. $(2011 b, 2012)$. Figure 1 displays the spatial distribution and $E\left(J-K_{\mathrm{s}}\right)$ colour excess for all 340 T2Cs and the histogram of colour excess is shown in the bottom panel. 
Table 1. Properties of Type II Cepheids in the Galactic bulge.

\begin{tabular}{|c|c|c|c|c|c|c|c|c|c|c|c|c|}
\hline \multirow[t]{2}{*}{ VVV ID } & \multirow[t]{2}{*}{ OGLE ID } & \multirow{2}{*}{$\begin{array}{c}P \\
\text { (days) }\end{array}$} & \multirow[t]{2}{*}{ Class } & \multicolumn{5}{|c|}{ Mean magnitudes } & \multicolumn{3}{|c|}{$\sigma$} & \multirow[t]{2}{*}{$E_{J K_{\mathrm{s}}}$} \\
\hline & & & & $V$ & $I$ & $J$ & $H$ & $K_{\mathrm{S}}$ & $J$ & $H$ & $K_{\mathrm{S}}$ & \\
\hline 515601356315 & OGLE-002 & 2.268 & BLH & 15.188 & 13.909 & 13.116 & 12.766 & 12.521 & 0.072 & 0.072 & 0.072 & 0.173 \\
\hline 515601679485 & OGLE-003 & 1.484 & BLH & 16.519 & 15.061 & 14.240 & 13.855 & 13.387 & 0.104 & 0.104 & 0.104 & 0.290 \\
\hline 515594023082 & OGLE-005 & 2.008 & BLH & 18.666 & 16.842 & 15.845 & 15.354 & 14.788 & 0.152 & 0.153 & 0.150 & 0.388 \\
\hline 515520862858 & OGLE-008 & 1.183 & BLH & 17.765 & 15.970 & - & 14.205 & 13.935 & - & 0.060 & 0.058 & 0.410 \\
\hline 515555436341 & OGLE-009 & 1.896 & BLH & 17.630 & 15.608 & 13.887 & 13.343 & 13.134 & 0.098 & 0.098 & 0.098 & 0.461 \\
\hline 515534145302 & OGLE-013 & 1.517 & BLH & 18.525 & 16.193 & 14.550 & 13.951 & 13.676 & 0.117 & 0.117 & 0.116 & 0.588 \\
\hline 515543870338 & OGLE-014 & 1.239 & BLH & 15.376 & 13.590 & 12.306 & 11.905 & 11.613 & 0.003 & 0.004 & 0.092 & 0.534 \\
\hline 515490128302 & OGLE-015 & 1.279 & BLH & 18.087 & 15.907 & 14.291 & 13.712 & 13.438 & 0.008 & 0.010 & 0.111 & 0.591 \\
\hline 515522173203 & OGLE-017 & 1.098 & BLH & 18.533 & 16.251 & 14.371 & 13.850 & 13.669 & 0.091 & 0.092 & 0.090 & 0.636 \\
\hline 515490198825 & OGLE-018 & 1.620 & BLH & 18.072 & 15.990 & 14.187 & 13.630 & 13.339 & 0.113 & 0.113 & 0.113 & 0.580 \\
\hline
\end{tabular}

Notes. The OGLE ID, period, subtype, and optical mean magnitudes are taken from OGLE-III (Soszyński et al. 2011). The values of $E_{J K_{\mathrm{s}}}$ : $E\left(J-K_{\mathrm{s}}\right)$, are taken from extinction maps of Gonzalez et al. (2011b). The first ten lines of the table are shown here and the entire table is available at the CDS.

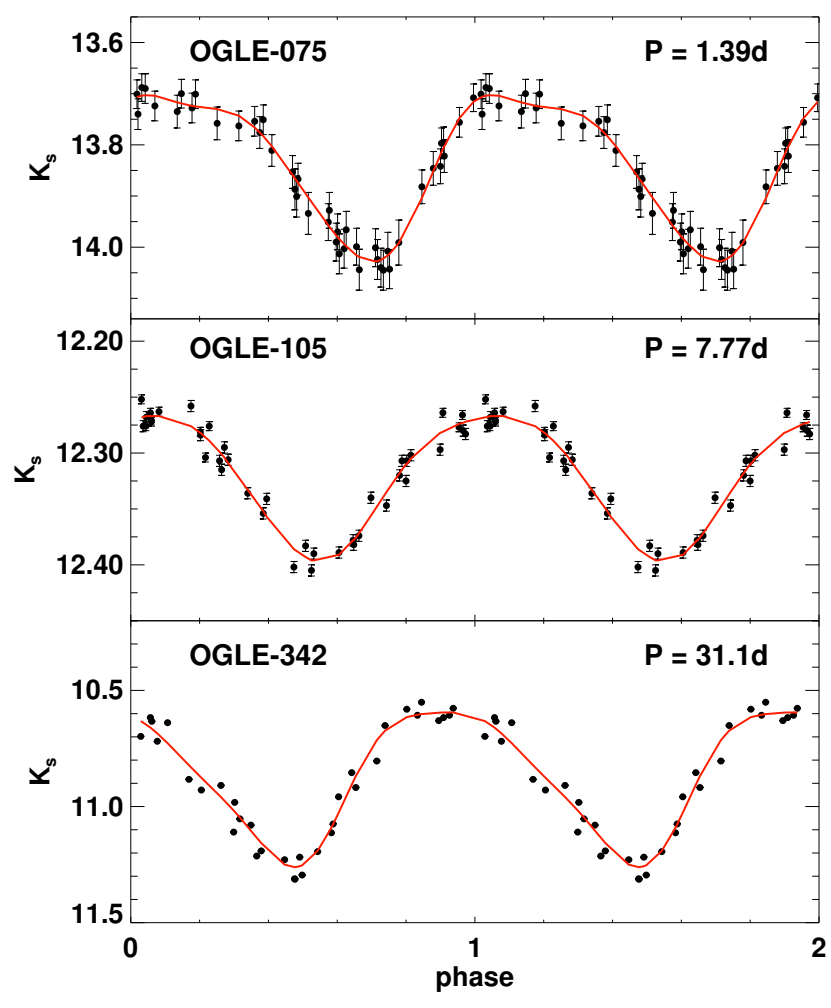

Fig. 2. Representative light curves in $K_{\mathrm{s}}$-band for Type II Cepheids in VVV survey. Top/middle/bottom panels display the BLH/WVR/RVT type variables. The solid red line is the Fourier fit to the light curve. Star IDs and periods are provided at the top of each panel.

In order to select the best-quality light curves, we only considered stars with a minimum of $20 K_{\mathrm{s}}$ measurements. We use periods and time of maximum brightness from OGLE to phase these light curves and apply a fifth order Fourier-series fit (see Bhardwaj et al. 2015) to determine the peak-to-peak amplitudes $(A)$ and the standard deviation $(\sigma)$ of the fit. We limit our final sample to the light curves for which $\sigma / A<1 / 20$. This provides an estimate of the impact that photometric uncertainties have on the shape of the light curves. Finally, we also remove four noisy light curves with very low-amplitudes $(A<0.08$ mag $)$. Following these selection criteria, we are left with a final sample of 264 T2Cs. Figure 2 displays the representative light curves of BLH,
WVR, and RVT stars from our final sample of good-quality light curves.

The saturation limit for $K_{\mathrm{s}}$-band in VVV survey is $\sim 11 \mathrm{mag}$ (see Fig. 2 in Gonzalez et al. 2011b) and the deviation from Two Micron All Sky Survey (2MASS) occurs around 12 mag in $J H$. While the higher extinction in the bulge can make these sources fainter, some of the long-period T2Cs may indeed be affected by non-linearity and saturation. We note that most of the rejected light-curves belong to long-period bright RVT stars and therefore this subclass will not be used for PLRs and distance estimates.

\section{Period-luminosity relations for Type II Cepheids in the bulge}

The mean-magnitudes in $K_{\mathrm{s}}$-band are estimated from the multiepoch VVV data by fitting templates from Bhardwaj et al. (2017) for T2Cs. For each single-epoch $J H$ measurement, we estimate the phase using the time of maximum brightness in $I$-band from the OGLE survey. We use $I K_{\mathrm{s}}$-band templates and apply phase correction to $J$ - and $H$-band magnitudes to derive their mean values. The mean properties of T2Cs in our final sample from OGLE and VVV are listed in Table 1.

Several studies have addressed the reddening law towards the Galactic centre (e.g. Nishiyama et al. 2006, 2009; Gonzalez et al. 2012; Nataf et al. 2016; Majaess et al. 2016) and its impact on the distance estimates will be discussed in the next section. For now, we adopt the Nishiyama et al. (2009) reddening law and total-to-selective absorption ratios, $R_{J}=1.526$, $R_{H}=0.855$, and $R_{K}=0.528$ corresponding to $E\left(J-K_{\mathrm{s}}\right)$, to apply extinction corrections. The $E\left(J-K_{\mathrm{s}}\right)$ value is greater than one for only 11 Cepheids in our sample. We also include reddening independent optical Wesenheit, $W_{V, I}=I-R_{I}^{V}(V-I)$, where the absorption-ratio, $R_{I}^{V}=1.08$, is adopted from Soszyński et al. (2011). The value of $R_{I}^{V}$ changes significantly depending on the choice of reddening law but we follow the OGLE relation for a relative comparison under the assumption that it exhibits the least scatter in the optical Wesenheit. We assume that all T2Cs are at the same distance and fit a PLR in the following form:

$m_{\lambda}^{0}=a_{\lambda}[\log (P)-1]+b_{\lambda}$,

where $m_{\lambda}^{0}$ is the extinction-corrected mean magnitude from the VVV survey and $\lambda$ represents the $J H K_{\mathrm{s}}$ wavelengths. The coefficient $a$ is the slope and $b$ is the zeropoint at $P=10 \mathrm{~d}$. We fit this equation to the BLH and WVR classes separately and 


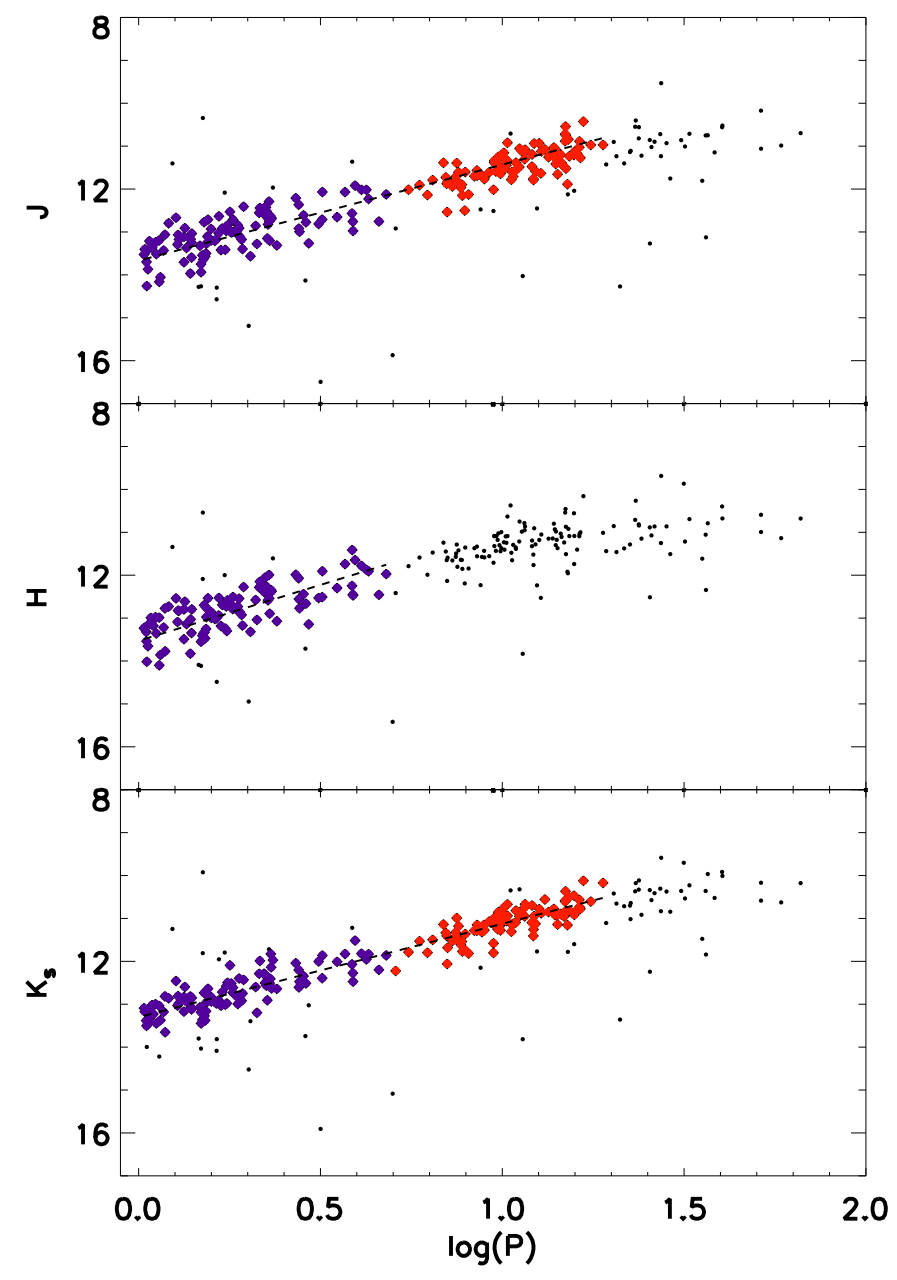

Fig. 3. Extinction-corrected NIR PLRs for Type II Cepheids in the Galactic bulge. The violet and red symbols represent BLH and WVR stars, respectively. The dashed line represents a single regression line fit over coloured symbols and small symbols represent the Type II Cepheids displaying evidence of approaching saturation in the long period range and $2 \sigma$ outliers in the short-period range. $H$-band data for WVR stars are also close to saturation limit and therefore we do not include them in linear regression fit.

also to the combined sample of BLH+WVR and iteratively remove $2 \sigma$ outliers in all cases. The adopted threshold provides a stronger constraint on the slopes and zeropoints of the PLRs and will be followed throughout the paper. We note that a higher sigma-clipping does not change the PLRs significantly and the number of stars and the dispersion increases marginally. Figure 3 displays the PLRs for T2Cs in the Galactic bulge. We observe a flattening of the $H$-band PLR for WVR variables. After looking at several $H$-band images, we find that these stars show evidence of approaching saturation. Therefore, we will use only BLH type variables in the $H$-band for the present analysis. The slopes and intercepts of PLRs are listed in Table 2. The dispersion in PLR is presumably dominated by the depth of the bulge with possible contribution due to the inner Galactic bar (Nishiyama et al. 2005; Gonzalez et al. 2011a).

We compare the Galactic bulge PLRs for T2Cs with published work in the Galactic globular clusters, the bulge, and the Large Magellanic Cloud (LMC) from Matsunaga et al. (2006), Groenewegen et al. (2008), and Bhardwaj et al. (2017). We note that Bhardwaj et al. (2017) derived new PLRs for T2Cs in the LMC and found the slopes and intercepts to be consistent with

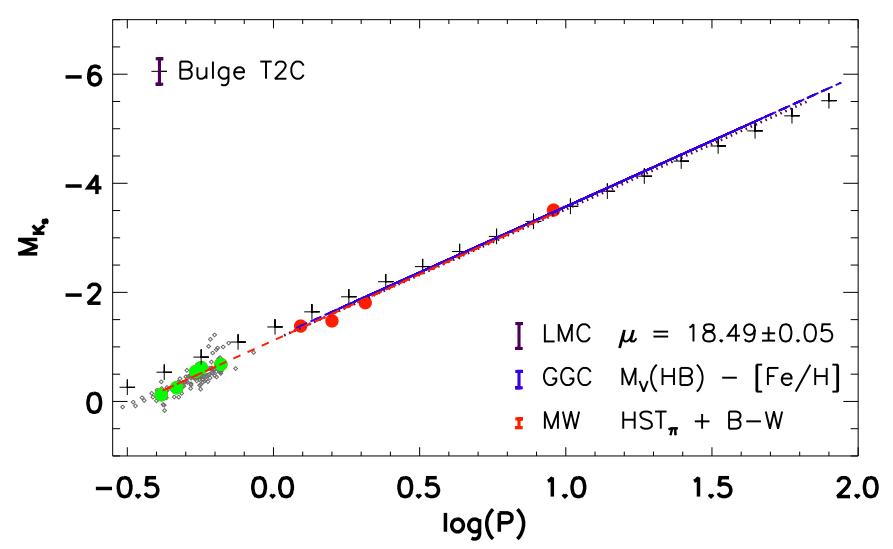

Fig. 4. Calibrated PLRs for Type II Cepheids in the LMC, Galactic globular clusters (GGC) and the Milky Way (solar neighbourhood). The red and green circles represent absolute magnitudes for T2Cs and RRLs with parallaxes in the Milky Way and the dashed red line is the bestfit linear regression. Plus symbols display PLR for VVV T2Cs with a zeropoint offset with respect to calibrated magnitudes. The error bars represent $1 \sigma$ dispersion in the PLR in each galaxy. Grey dots display absolute magnitudes for RRLs in the LMC and globular clusters.

Table 2. Galactic bulge Type II Cepheid PLRs.

\begin{tabular}{cccccc}
\hline \hline Band & Type & Slope & Intercept & $\sigma$ & $N$ \\
\hline$W_{V, I}$ & B $+\mathrm{W}$ & $-2.294 \pm 0.055$ & $11.511 \pm 0.030$ & 0.342 & 212 \\
$J$ & BLH & $-2.387 \pm 0.164$ & $11.393 \pm 0.132$ & 0.347 & 106 \\
$J$ & WVI & $-2.037 \pm 0.096$ & $11.476 \pm 0.012$ & 0.242 & 93 \\
$J$ & B+W & $-2.240 \pm 0.031$ & $11.495 \pm 0.009$ & 0.316 & 203 \\
$H$ & BLH & $-2.591 \pm 0.163$ & $11.019 \pm 0.130$ & 0.353 & 104 \\
$K_{\mathrm{s}}$ & BLH & $-2.362 \pm 0.170$ & $11.071 \pm 0.133$ & 0.294 & 108 \\
$K_{\mathrm{s}}$ & WVI & $-2.373 \pm 0.272$ & $11.194 \pm 0.034$ & 0.194 & 95 \\
$K_{\mathrm{S}}$ & B+W & $-2.189 \pm 0.056$ & $11.187 \pm 0.032$ & 0.234 & 201 \\
\hline
\end{tabular}

Notes. B+W: BL Herculis and W Virginis.

previous results. Therefore, we only consider PLRs in the LMC from Bhardwaj et al. (2017). We also compare the optical Wesenheit in the bulge with LMC, where the total-to-selective absorption ratio, $R_{I}^{V}=1.55$, is taken from Soszyński et al. (2010) for LMC T2Cs. We note that optical Wesenheits for T2Cs in the LMC and bulge are adopted only for a relative comparison and these relations will not be used for distance estimates. We will use a standard t-test to compare the PLRs, given the uncertainties in the slopes and the rms. of the relation under consideration. The details of the test statistics are discussed in Bhardwaj et al. (2016). In brief, the null hypothesis, that is, that the two slopes are equal, is rejected if the probability $p(t)<0.05$.

The results of t-test statistics are listed in Table 3 . We find that the $W_{V, I}$ Wesenheits and $K_{\mathrm{s}}$-band PLRs in the Galactic bulge and the LMC are consistent, if we consider only BLH+WVR variables. The slope of the $K_{\mathrm{s}}$-band PLR is consistent with the result of Groenewegen et al. (2008) for the bulge, while there is a marginal but statistically significant difference in slopes with Galactic globular clusters. We also compare the $K_{\mathrm{s}}$-band PLRs with the calibrated PLRs for T2Cs and RRLs using Gaia and Hubble Space Telescope (HST) parallaxes from Bhardwaj et al. (2017). The PLRs are consistent, given the large uncertainties in the slope of the calibrated PLR.

Figure 4 displays the calibrated PLRs in the LMC (violet), Galactic globular clusters (blue), and the Milky Way (MW, red). We adopt the late-type eclipsing binary distance 
Table 3. Comparison of Galactic bulge Type II Cepheids with PLRs from literature.

\begin{tabular}{ccccccccc}
\hline \hline Band & Slope & $\sigma$ & $N$ & Host & Type & Source & $|T|$ & $p(t)$ \\
\hline$W_{V, I}$ & $-2.294 \pm 0.055$ & 0.342 & 212 & GB & B+W & TW & - & - \\
& $-2.677 \pm 0.052$ & 0.176 & 186 & LMC & all & TW & 4.265 & 0.000 \\
& $-2.508 \pm 0.074$ & 0.111 & 133 & LMC & B+W & TW & 1.171 & 0.242 \\
$K_{\mathrm{S}}$ & $-2.189 \pm 0.056$ & 0.234 & 201 & GB & B + W & TW & - & - \\
& $-2.240 \pm 0.140$ & 0.410 & 39 & GB & all & G08 & 0.462 & 0.644 \\
& $-2.395 \pm 0.027$ & 0.228 & 167 & LMC & all & B17 & 3.621 & 0.000 \\
& $-2.232 \pm 0.037$ & 0.180 & 119 & LMC & B+W & B17 & 0.648 & 0.517 \\
& $-2.413 \pm 0.053$ & 0.150 & 43 & GGC & all & M06 & 2.309 & 0.022 \\
& $-2.425 \pm 0.295$ & 0.075 & 9 & MW & all & B17 & 0.243 & 0.808 \\
\hline
\end{tabular}

Notes. B+W: BL Herculis and W Virginis; ${ }^{(a)}$ represents absolute calibration of PLRs based on parallaxes for T2Cs and RRLs. Source column represents - TW: this work; G08: Groenewegen et al. (2008); B17: Bhardwaj et al. (2017); M06: Matsunaga et al. (2006) PLR with updated mean magnitudes from Bhardwaj et al. (2017). Last two columns represent the observed value of the $t$-statistic $(|T|)$ and the probability, $p(t)$, of acceptance of the null hypothesis.

of $18.493 \pm 0.047$ mag (Pietrzyński et al. 2013) to calibrate LMC PLR. The two T2Cs and five RRLs in the solar neighbourhood are calibrated with available trigonometric parallaxes from Gaia and HST (see, Bhardwaj et al. 2017). We also include two T2Cs with pulsation parallaxes from Feast et al. (2008). We note that the entire period-range $K_{\mathrm{s}}$-band PLRs in the LMC, Galactic globular cluster, and in the MW have nearly the same slopes and zeropoints. This provides additional evidence that $K_{\mathrm{s}}$-band PLRs are less sensitive to the metallicity and extinction and can be used to obtain accurate distance estimates. The $K_{\mathrm{s}}$ mean magnitudes for RRLs are also included to extend the PLRs followed by T2Cs (see grey symbols in Fig. 4). The NIR photometry of RRLs in the LMC is taken from Borissova et al. (2009) and Muraveva et al. (2015), while the globular clusters RRLs data is adopted from Messier 92 and Messier 4 (Del Principe et al. 2005; Stetson et al. 2014). This further confirms the consistency between the distance scale for T2Cs and RRLs as discussed in previous papers (Sollima et al. 2006; Matsunaga et al. 2006; Ripepi et al. 2015; Bhardwaj et al. 2017).

\section{Distance to the Galactic centre}

We use $K_{\mathrm{s}}$-band mean magnitudes for T2Cs in the Galactic bulge to determine a distance to the Galactic centre using the absolute calibration of PLR for T2Cs and RRLs in the MW and in the LMC. We only use short period BLH and WVR stars and apply separate calibrations based on MW and LMC T2C PLRs to the bulge data to obtain two sets of individual distances. We take an average of the two distances for each BLH+WVR type variables in $K_{\mathrm{s}}$-band. Figure 5 displays the histogram of individual distance estimates for BLH+WVR and RRLs variables. We include OGLE-IV counterparts of the RRLs from the VVV survey in our analysis for a relative comparison. The RRLs sample consists of more than 20 thousand variables with high-quality light curves and their photometry will be discussed in a future publication.

To determine the distance to the centre of the population, we need to apply two geometric corrections. First, the individual distances are projected onto the Galactic plane. This is done by taking the cosine of the Galactic latitude, resulting in a distance of Rcosb. Secondly, we need to correct the distance distribution for the "cone-effect", which leads to bias in distance estimates as more objects are observed at larger distances in a solid angle. This is corrected by scaling the distance distribution by $R^{-2}$. The two histogram bars in Fig. 5 represent the distances before (blue) and after (magenta) these corrections. In our sample the
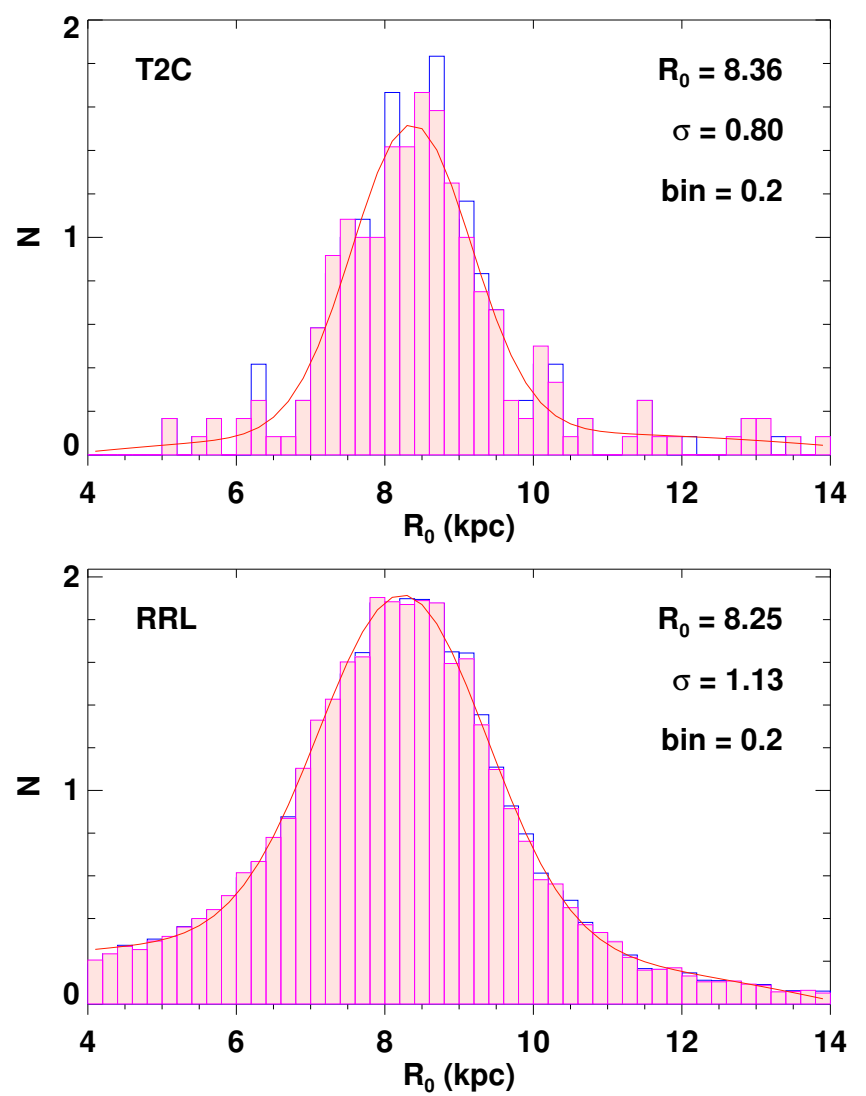

Fig. 5. Top panel: histogram of the projected distances of BLH+WVR T2Cs. The blue and magenta bars represent the distance distribution before and after the geometric corrections. Bottom panel: same as top panel but for RRLs in the VVV survey.

correction is marginal $(\sim 0.5 \%)$ in terms of the change in the peak of the distance distribution. We note that both T2Cs and RRLs provide similar estimates for the distance to the Galactic centre. The RRLs distance distribution is based on empirical calibration of T2C PLR and does not include any metallicity corrections.

In order to obtain a robust distance estimate, we use $K_{\mathrm{s}}$-band mean magnitudes for the T2Cs and apply a simultaneous fit to all BLH+WVR stars in the Galactic bulge, LMC, Galactic globular clusters, and T2Cs in the solar neighbourhood. We fit a PLR in the following form:

$m_{i j}^{0}=a_{\mathrm{S}}\left[\log \left(P_{i j}\right)-1\right]+a_{L}\left[\log \left(P_{i j}\right)-1\right]+M_{K_{\mathrm{S}}}+\mu_{j}$, 

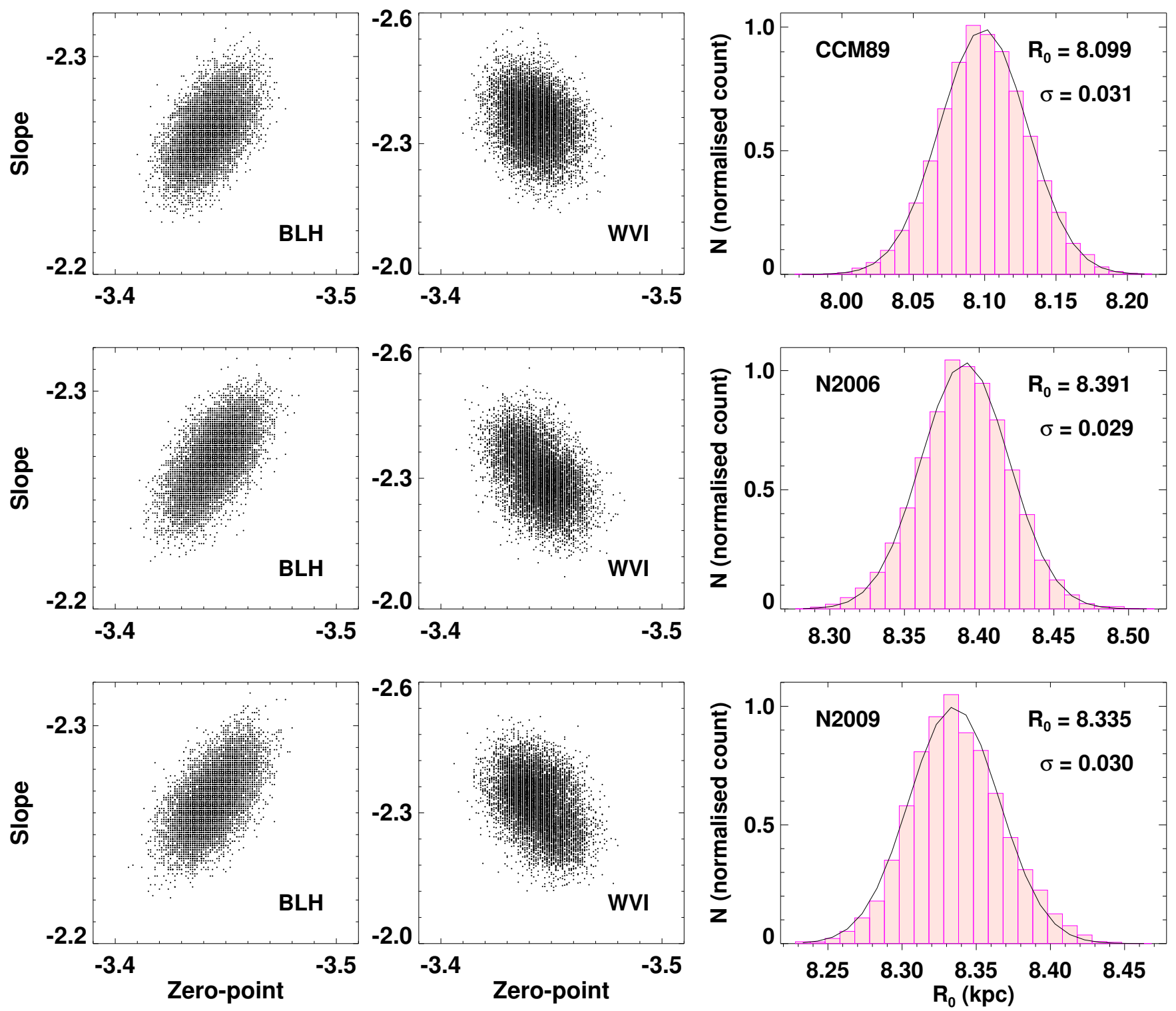

Fig. 6. Result of $10^{4}$ random realizations of parameters of the global fit to Type II (BLH+WVR) Cepheids in the globular clusters, LMC, and the Galactic bulge. Left and middle panels: slope and the zeropoint of global fit. Right panels: distance estimates from 10 4 permutations. CCM89: Cardelli et al. (1989); N2006: Nishiyama et al. (2006); N2009: Nishiyama et al. (2009).

where $m_{i j}^{0}$ is the extinction-corrected magnitude for a $i$ th $\mathrm{T} 2 \mathrm{C}$ in the $j$ th system. The coefficients $a_{\mathrm{S}}$ and $a_{L}$ represent the slopes of BLH and WVR stars and $M_{K_{\mathrm{s}}}$ is the absolute magnitude in $K_{\mathrm{s}}$-band for a T2C with $P=10$ days. The distance modulus to the Galactic bulge is given by $\mu_{j}$. The matrix equation is solved using the chi-square minimization (Bhardwaj et al. 2016).

Nishiyama et al. (2009) and Matsunaga et al. (2016, and references therein) showed that the selection of reddening law leads to a significant difference in distance estimates close to the Galactic centre. Therefore, we use three different extinction laws, $\frac{A_{K_{\mathrm{s}}}}{E\left(J-K_{\mathrm{s}}\right)}=0.689$ (Cardelli et al. 1989), $\frac{A_{K_{\mathrm{s}}}}{E\left(J-K_{\mathrm{s}}\right)}=0.494$ (Nishiyama et al. 2006), and $\frac{A_{K_{\mathrm{s}}}}{E\left(J-K_{\mathrm{s}}\right)}=0.528$ (Nishiyama et al. 2009) in our analysis.

We carry out Monte-Carlo simulations to create $10^{4}$ random realizations of the global fit. We estimate the coefficients of the global fit for each permutation and fit a Gaussian function to the histograms to determine a mean value with their statistical uncertainties. Figure 6 displays the variation of $10^{4}$ random
Table 4. Parameters of the global fit.

\begin{tabular}{cccc}
\hline \hline & CCM89 & N2006 & N2009 \\
\hline$a_{\mathrm{S}}$ & $-2.261 \pm 0.013$ & $-2.266 \pm 0.014$ & $-2.261 \pm 0.014$ \\
$a_{L}$ & $-2.354 \pm 0.062$ & $-2.299 \pm 0.068$ & $-2.328 \pm 0.066$ \\
$M_{K_{\mathrm{S}}}$ & $-3.438 \pm 0.010$ & $-3.441 \pm 0.011$ & $-3.439 \pm 0.010$ \\
$\mu$ & $14.552 \pm 0.008$ & $14.630 \pm 0.008$ & $14.616 \pm 0.008$ \\
$R_{0}$ & $8.099 \pm 0.031$ & $8.391 \pm 0.029$ & $8.335 \pm 0.030$ \\
\hline
\end{tabular}

Notes. CCM89: Cardelli et al. (1989); N2006: Nishiyama et al. (2006); N2009: Nishiyama et al. (2009).

realizations of the slopes and the zeropoint (left and middle panel) and the histogram of distance estimates (right panel) for three different extinction laws. The coefficients of the global fit are given in Table 4. The slope of WVR type variables shows a greater variation as compared to BLH type variables. As expected, due to similar extinction coefficients, the difference 


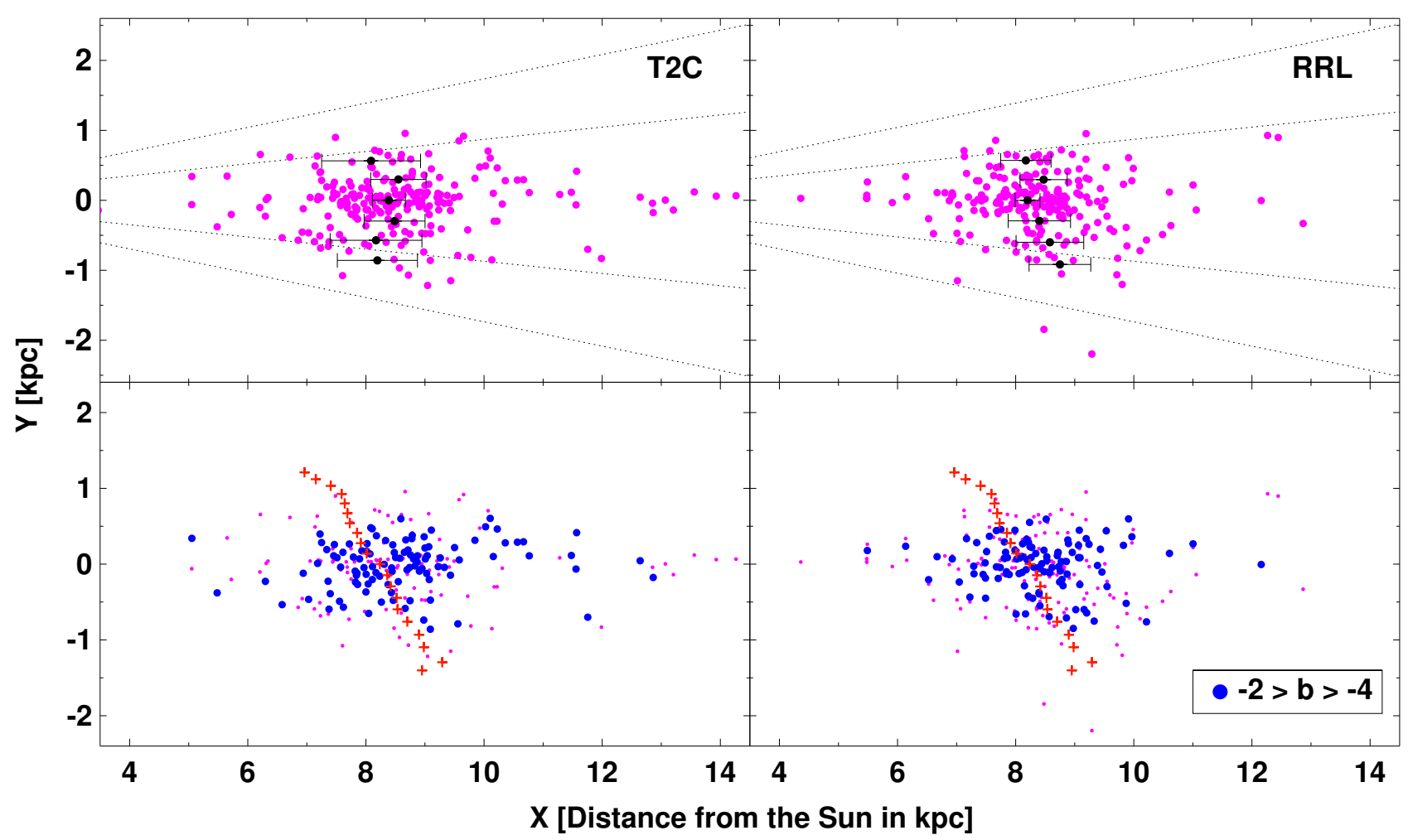

Fig. 7. Top panels: spatial distribution of T2Cs (left column) and RRLs (right column) projected onto the Galactic plane. RRLs are drawn for each representative T2C from the parent sample of RRLs in the VVV survey. The black circles represent projected mean line-of-sight distances in different longitude bins and error bars display $3 \sigma$ standard deviation of the mean. The dotted lines represent the line of sight corresponding to $l= \pm 5$ and $l= \pm 10$. Bottom panels: the spatial distribution of T2Cs and RRLs in the latitude stripe $-2>b>-4$ (large blue dots) and outside these latitudes (small magenta dots) are compared with the mean red clump giants' distances (red plus symbols). The mean distances to red clump giants are derived from peaks of Gaussian distributions in each 1 sq. deg. field for latitudes in the range $-2>b>-4$ (Gonzalez et al. 2012 ; Valenti et al. 2016), and assuming a mean absolute magnitude $M_{K}=-1.61 \pm 0.015$ (Laney et al. 2012).

between distance estimates from the two Nishiyama extinction laws is not significant, but the distance to Galactic centre differs significantly when compared to the distance obtained using Cardelli et al. (1989). We adopt the distance to the Galactic centre, $R_{0}=8.34 \pm 0.03 \mathrm{kpc}$, obtained using the reddening law of Nishiyama et al. (2009). This value is close to the value obtained using the Nishiyama et al. (2006) reddening law, but is more consistent with recent distance estimates from other distance indicators, such as RRLs from the VVV survey, $R_{0}=8.33 \pm 0.05$ (stat.) \pm 0.14 (syst.) kpc (Dékány et al. 2013) and from OGLE-IV, $R_{0}=8.27 \pm 0.01$ (stat.) \pm 0.40 (syst.) kpc (Pietrukowicz et al. 2015). Our distance to the Galactic centre is in excellent agreement with the recommended distance, $R_{0}=8.3 \pm 0.2$ (stat.) \pm 0.4 (syst.) kpc, by de Grijs \& Bono (2016). We note that these distance estimates are also consistent with results based on studies of stellar orbits in the Galactic centre and distance to central black hole $\left(R_{0}=8.32 \pm 0.07\right.$ (stat. $) \pm$ 0.14(syst.) kpc, Genzel et al. 2010; Gillessen et al. 2017, and references therein). We adopt a conservative approach for the systematic uncertainty on the distance to the Galactic centre and include the error on the photometry (median $\sim 0.08 \mathrm{mag}$ ), the error on the $A_{K_{\mathrm{s}}}$ extinction values (median $\sim 0.07 \mathrm{mag}$ ), uncertainty in the zeropoint of the calibrated PLRs (0.02 mag, inverse weighted variance resulting from two independent calibrations), which amounts to $\sim 0.11 \mathrm{mag}(0.41 \mathrm{kpc})$.

\section{The spatial distribution of population II Cepheids}

The distribution of old metal-poor stellar populations unveils an axisymmetric component of the bulge, while metal-rich red-clump giants show an elongated distribution that traces the bar (Gonzalez et al. 2011b; Zoccali et al. 2017). Metal-poor RRLs, Miras, and red-clump giants display an axisymmetric and spheroidal structure of the bulge (Dékány et al. 2013; Catchpole et al. 2016; Zoccali et al. 2017). RRLs also show a triaxial ellipsoid shape with OGLE-IV data (Pietrukowicz et al. 2015). We look at the spatial distribution of T2Cs projected onto the Galactic plane. We also select a representative RRL within a $1^{\prime}$ radius of each $\mathrm{T} 2 \mathrm{C}$ position from the distribution of RRLs shown in Fig. 5 and adopt a median distance.

The top panel of Fig. 7 displays the spatial distribution of $\mathrm{T} 2 \mathrm{Cs}$, projected onto the Galactic plane. The T2C sample shows a homogeneous distribution with the majority of distance estimates falling within $6-10 \mathrm{kpc}$. The corresponding RRL distribution is more spherically symmetric. The T2C distance distribution shows more stars at longer distances as compared to the RRL subsample that is slightly elongated towards us. We also overplot mean line-of-sight distances in different longitude bins for T2Cs and RRLs. Neither population provides evidence of an inclined bar or X-shaped structure as traced by the metal-rich red-clump giants. We note that the majority of T2Cs in the current sample are located along $b=-2$ to $b=-4$ and their spatial distribution is shown in the bottom panel. The distribution along these latitudes confirms a no-barred distribution with current T2C and RRL samples in the bulge.

We also performed a two-sided Kolmogorov-Smirnov test to compare the distance distributions of T2Cs and RRLs from the VVV survey. We find that both these populations have similar radial distributions in most spatial bins. However, the number density of T2Cs is very limited at present and the results of test statistics are heavily influenced by small number statistics and 
the choice of bin size. In the near future, with more data from OGLE-IV and VVV, T2Cs could become additional important tracers of the bulge metal-poor old populations, probing structure and formation of the Galactic bulge.

We recall that our T2C and RRL distance estimates are based on $K_{\mathrm{s}}$-band mean magnitudes from the VVV survey. We do not use Wesenheit relations to get the distance estimates as the time-series observations are available only in $K_{\mathrm{s}}$-band. Furthermore, given that the $K_{\mathrm{s}}$-band PLR has the least dispersion (see Fig. 4 and Tables 2 and 3), we deem it is better to apply extinction correction to the $K_{\mathrm{s}}$-band PLR adopting external reddening values rather than to construct a Wesenheit with random-phase corrected $J H$ magnitudes. The extinction correction is applied using the Nishiyama et al. (2009) reddening law and $E\left(J-K_{\mathrm{s}}\right)$ colour excess values from the extinction maps of Gonzalez et al. (2011b, 2012), which are derived by comparing the mean $E\left(J-K_{\mathrm{s}}\right)$ colour of red clump giants in small subfields of $2^{\prime} \times 2^{\prime}$ to $6^{\prime} \times 6^{\prime}$ with the colour of red clumps in Baade's window (see Gonzalez et al. 2012, for details). The colour excess for each T2C and RRL is obtained by inserting their longitude and latitude into the Bulge Extinction and Metallicity Calculator $(\mathrm{BEAM})^{1}$ and adopting a resolution of $2^{\prime}$.

Given that the 2D BEAM extinction map assumes all extinction is at the location of the tracer (red clumps, which are mostly located in the bulge/bar), while our targets are located along the line of sight at different distances with respect to the bar, we compare the BEAM $E\left(J-K_{\mathrm{s}}\right)$ values with $E\left(J-K_{\mathrm{s}}\right)$ from the $3 \mathrm{D}$ extinction map of Schultheis et al. (2014). The 3D extinction maps are based on the VVV data and temperature-colour relation for $\mathrm{M}$ giants and the distance-colour relations. The stellar population synthesis models were used to offset observed and intrinsic colours and obtain extinction maps with two spatial and one distance dimension.

We compare the distance estimates for each T2C and RRL at a given longitude and latitude and obtain $E\left(J-K_{\mathrm{S}}\right)$ colour excess from $3 \mathrm{D}$ maps. The difference in $E\left(J-K_{\mathrm{s}}\right)$ colour excess values as a function of distance is shown in the top panel of Fig. 8 . The difference increases for the line-of-sight distances towards us and the offset is $\sim-0.05$ mag for T2Cs and $\sim-0.03$ mag for RRLs close to the Galactic centre. We also correct the extinction values from Gonzalez et al. (2012) and corresponding distance estimates by iteratively computing the difference in extinction with respect to $3 \mathrm{D}$ maps. The initial $E\left(J-K_{\mathrm{s}}\right)$ values are corrected for offset and the distances are redetermined in each iteration. The procedure is repeated until extinction or distance estimates converge.

The bottom panel of Fig. 8 shows the spatial distribution of T2Cs before (grey) and after (magenta) the extinction correction from 3D maps. At present, the accuracy of the distance dimension of 3D maps is limited to the bin size of $0.5 \mathrm{kpc}$ (up to $10.5 \mathrm{kpc}$ ) and therefore the correction in extinction converges typically in the second or third iteration. Thus, the difference in resulting distance distribution is very small and not statistically significant. Median difference in extinction is $\sim 0.01$ and the distance to the Galactic centre changes only marginally $\left(\sim 10^{-3}\right)$ if we use corrected extinctions from 3D maps.

\section{Conclusions}

We summarize our results as follows:

- We present a catalogue with mean VIJHK magnitudes, periods, reddening, and subclass classifications for 264 Type II

1 http://mill.astro.puc.cl/BEAM/calculator.php
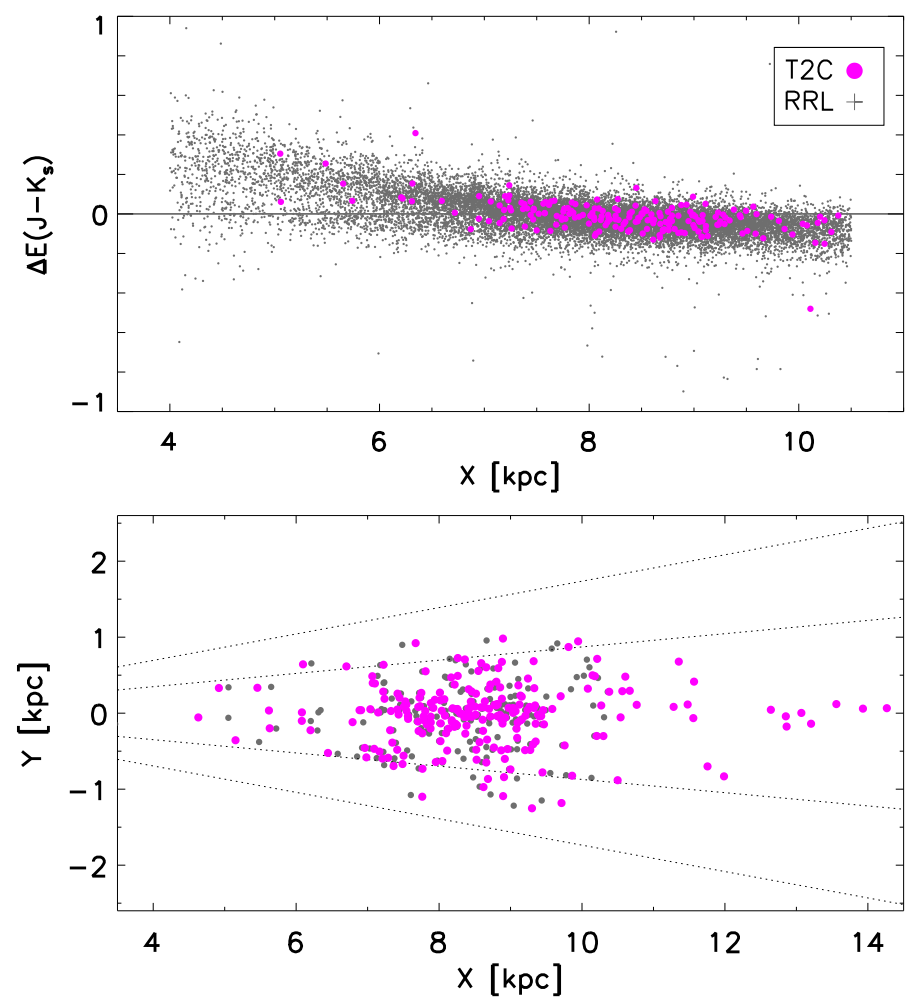

Fig. 8. Top panel: difference in $E\left(J-K_{\mathrm{s}}\right)$ values for T2Cs and RRLs obtained from extinction maps based on red clump giants (Gonzalez et al. 2012) and 3D extinction map of Schultheis et al. (2014) as a function of distance. Bottom panel: spatial distribution of T2Cs before (grey) and after (magenta) the extinction correction using 3D map.

Cepheids in the Galactic bulge by matching the VVV NIR observations with optical data from OGLE-III. The sample consists of various subtypes (BL Herculis, W Virginis, and RV Tauris) with an average of $50 K_{\mathrm{s}}$ measurements per light curve.

- We use random-phase corrected $J H$ magnitudes and $K_{\mathrm{s}}$ mean magnitudes to derive period-luminosity relations for Type II Cepheids. The long-period RV Tauris stars are affected by saturation and therefore a sample of BL Herculis and W Virginis stars is used in our distance analysis. The period-luminosity relation in $K_{\mathrm{s}}$-band, $K_{\mathrm{s}}=$ $-2.189(0.056)[\log (P)-1]+11.187(0.032)$, is found to be consistent with published work for the LMC variables.

- We apply a global fit to the Galactic bulge, LMC, and Galactic globular cluster Type II Cepheid data in $K_{\mathrm{s}}$-band, together with calibrated absolute magnitudes for Type II Cepheids and RR Lyrae with Gaia and Hubble Space Telescope parallaxes, to determine a distance to the Galactic centre, $R_{0}=$ $8.34 \pm 0.03$ (stat.) \pm 0.41 (syst.) kpc. Our results are in a very good agreement with published distance measurements based on Type II Cepheids, RRLs (Groenewegen et al. 2008; Dékány et al. 2013; Pietrukowicz et al. 2015), and the recommended distance by de Grijs \& Bono (2016). Adopting a different extinction law amounts to a difference of ${ }_{-0.25}^{+0.05}$ in the final distance to Galactic centre.

- We also investigated the spatial distribution of Type II Cepheids in the Galactic bulge. We compared their distribution with well-studied most abundant tracers in the bulge, such as RR Lyrae and red-clump giants. We find that Type II Cepheids display a non-barred distribution, similar to other metal-poor bulge tracers, RR Lyrae (Dékány et al. 2013), and 


\section{A. Bhardwaj et al.: Type II Cepheids in the Galactic bulge}

red-clump giants (Zoccali et al. 2017). This result requires further validation with a larger sample of T2Cs that are uniformly distributed, in particular at low latitudes.

- We test the individual distance estimates and extinction values for Type II Cepheids in the Galactic bulge by taking into account the 3D distribution of dust in the Milky Way. At present, considering the discretization of 3D maps along distance dimension in steps of $0.5 \mathrm{kpc}$, the correction of individual distances is not significant.

Acknowledgements. A.B. is thankful to the Council of Scientific and Industrial Research, New Delhi, for a Senior Research Fellowship (SRF, 09/045(1296)/2013-EMR-I). We acknowledge the use of data from the ESO Public Survey programme ID 179.B-2002 taken with the VISTA telescope. D.M., M.R., E.V., M.Z., and O.G. acknowledge the hospitality of the Aspen Center for Physics, where this work was initiated. The Aspen Center for Physics is supported by National Science Foundation grant PHY-1066293. D.M. and M.Z. were partially supported by a grant from the Simons Foundation during their stay in Aspen and gratefully acknowledge support by FONDECYT Regular grant Nos. 1130196 and 1150345, by the Ministry of Economy, Development and Tourism's Millennium Science Initiative through grant IC120009, awarded to The Millennium Institute of Astrophysics (MAS) and by the BASAL-CATA Center for Astrophysics and Associated Technologies PFB-06.

\section{References}

Alcock, C., Allsman, R. A., Alves, D. R., et al. 1998, AJ, 115, 1921 Bensby, T., Feltzing, S., Gould, A., et al. 2017, A\&A, 605, A89 Bhardwaj, A., Kanbur, S. M., Singh, H. P., Macri, L. M., \& Ngeow, C.-C. 2015 , MNRAS, 447, 3342

Bhardwaj, A., Kanbur, S. M., Macri, L. M., et al. 2016, AJ, 151, 88

Bhardwaj, A., Macri, L. M., Rejkuba, M., et al. 2017, AJ, 153, 154

Borissova, J., Rejkuba, M., Minniti, D., Catelan, M., \& Ivanov, V. D. 2009, A\&A, 502, 505

Cardelli, J. A., Clayton, G. C., \& Mathis, J. S. 1989, ApJ, 345, 245

Catchpole, R. M., Whitelock, P. A., Feast, M. W., et al. 2016, MNRAS, 455 2216

Ciechanowska, A., Pietrzyński, G., Szewczyk, O., Gieren, W., \& Soszyński, I. 2010, Acta Astron., 60, 233

Clarkson, W., Sahu, K., Anderson, J., et al. 2008, ApJ, 684, 1110

Clarkson, W. I., Sahu, K. C., Anderson, J., et al. 2011, ApJ, 735, 37

de Grijs, R., \& Bono, G. 2016, ApJS, 227, 5

Dékány, I., Minniti, D., Catelan, M., et al. 2013, ApJ, 776, L19

Del Principe, M., Piersimoni, A. M., Bono, G., et al. 2005, AJ, 129, 2714

Feast, M. W., Laney, C. D., Kinman, T. D., van Leeuwen, F., \& Whitelock, P. A. 2008, MNRAS, 386, 2115

Genzel, R., Eisenhauer, F., \& Gillessen, S. 2010, Rev. Mod. Phys., 82, 3121

Gillessen, S., Plewa, P. M., Eisenhauer, F., et al. 2017, ApJ, 837, 30

Gonzalez, O. A., Rejkuba, M., Minniti, D., et al. 2011a, A\&A, 534, L14

Gonzalez, O. A., Rejkuba, M., Zoccali, M., Valenti, E., \& Minniti, D. 2011b, A\&A, 534, A3
Gonzalez, O. A., Rejkuba, M., Zoccali, M., et al. 2012, A\&A, 543, A13 Gonzalez, O. A., Rejkuba, M., Zoccali, M., et al. 2013, A\&A, 552, A110 Gonzalez, O. A., Zoccali, M., Debattista, V. P., et al. 2015a, A\&A, 583, L5 Gonzalez, O. A., Zoccali, M., Vasquez, S., et al. 2015b, A\&A, 584, A46 Gran, F., Minniti, D., Saito, R. K., et al. 2016, A\&A, 591, A145 Groenewegen, M. A. T., Udalski, A., \& Bono, G. 2008, A\&A, 481, 441 Hill, V., Lecureur, A., Gómez, A., et al. 2011, A\&A, 534, A80

Kubiak, M., \& Udalski, A. 2003, Acta Astron., 53, 117

Kuijken, K., \& Rich, R. M. 2002, AJ, 124, 2054

Laney, C. D., Joner, M. D., \& Pietrzyński, G. 2012, MNRAS, 419, 1637

Leavitt, H. S., \& Pickering, E. C. 1912, Harvard College Observatory Circular, 173,1

Majaess, D., Turner, D., \& Lane, D. 2009, Acta Astron., 59, 403

Majaess, D., Turner, D., Dékány, I., Minniti, D., \& Gieren, W. 2016, A\&A, 593, A124

Matsunaga, N., Fukushi, H., Nakada, Y., et al. 2006, MNRAS, 370, 1979

Matsunaga, N., Feast, M. W., \& Menzies, J. W. 2009, MNRAS, 397, 933

Matsunaga, N., Feast, M. W., \& Soszyński, I. 2011, MNRAS, 413, 223

Matsunaga, N., Feast, M. W., Bono, G., et al. 2016, MNRAS, 462, 414

McWilliam, A., \& Zoccali, M. 2010, ApJ, 724, 1491

Minniti, D., Lucas, P. W., Emerson, J. P., et al. 2010, New Astron., 15, 433

Minniti, D., Contreras Ramos, R., Zoccali, M., et al. 2016, ApJ, 830, L14

Muraveva, T., Palmer, M., Clementini, G., et al. 2015, ApJ, 807, 127

Nataf, D. M., Udalski, A., Gould, A., Fouqué, P., \& Stanek, K. Z. 2010, ApJ, 721, L28

Nataf, D. M., Gonzalez, O. A., Casagrande, L., et al. 2016, MNRAS, 456, 2692

Nemec, J. M., Nemec, A. F. L., \& Lutz, T. E. 1994, AJ, 108, 222

Ness, M., Freeman, K., Athanassoula, E., et al. 2013, MNRAS, 430, 836

Nishiyama, S., Nagata, T., Baba, D., et al. 2005, ApJ, 621, L105

Nishiyama, S., Nagata, T., Kusakabe, N., et al. 2006, ApJ, 638, 839

Nishiyama, S., Tamura, M., Hatano, H., et al. 2009, ApJ, 696, 1407

Ortolani, S., Renzini, A., Gilmozzi, R., et al. 1995, Nature, 377, 701

Pietrukowicz, P., Kozłowski, S., Skowron, J., et al. 2015, ApJ, 811, 113

Pietrzyński, G., Graczyk, D., Gieren, W., et al. 2013, Nature, 495, 76

Ripepi, V., Moretti, M. I., Marconi, M., et al. 2015, MNRAS, 446, 3034

Rojas-Arriagada, A., Recio-Blanco, A., Hill, V., et al. 2014, A\&A, 569, A103

Sahu, K. C., Casertano, S., Bond, H. E., et al. 2006, Nature, 443, 534

Saito, R. K., Zoccali, M., McWilliam, A., et al. 2011, AJ, 142, 76

Saito, R. K., Hempel, M., Minniti, D., et al. 2012, A\&A, 537, A107

Sandage, A., \& Tammann, G. A. 2006, ARA\&A, 44, 93

Schmidt, E. G., Hemen, B., Rogalla, D., \& Thacker-Lynn, L. 2009, AJ, 137, 4598

Schultheis, M., Chen, B. Q., Jiang, B. W., et al. 2014, A\&A, 566, A120

Sollima, A., Cacciari, C., \& Valenti, E. 2006, MNRAS, 372, 1675

Soszyński, I., Udalski, A., Szymański, M. K., et al. 2008, Acta Astron., 58, 293

Soszyński, I., Udalski, A., Szymański, M. K., et al. 2010, Acta Astron., 60, 91

Soszyński, I., Udalski, A., Pietrukowicz, P., et al. 2011, Acta Astron., 61, 285

Stetson, P. B., Braga, V. F., Dall'Ora, M., et al. 2014, PASP, 126, 521

Valenti, E., Zoccali, M., Renzini, A., et al. 2013, A\&A, 559, A98

Valenti, E., Zoccali, M., Gonzalez, O. A., et al. 2016, A\&A, 587, L6

Wallerstein, G. 2002, PASP, 114, 689

Wegg, C., \& Gerhard, O. 2013, MNRAS, 435, 1874

Zoccali, M., \& Valenti, E. 2016, PASA, 33, e025

Zoccali, M., Renzini, A., Ortolani, S., et al. 2003, A\&A, 399, 931

Zoccali, M., Hill, V., Lecureur, A., et al. 2008, A\&A, 486, 177

Zoccali, M., Vasquez, S., Gonzalez, O. A., et al. 2017, A\&A, 599, A12 